\title{
The Power of Exercise-Induced T-wave Alternans to Predict Ventricular Arrhythmias in Patients with Implanted Cardiac Defibrillator
}

\author{
Laura Burattini, $\mathrm{PhD}^{1 *}$, Sumche Man, $\mathrm{MD}^{2}$ and Cees $\mathrm{A}$. Swenne, $\mathrm{PhD}, \mathrm{MD}^{2}$ \\ ${ }^{I}$ Department of Information Engineering, \\ Polytechnic University of Marche, Ancona, Italy. \\ ${ }^{2}$ Department of Cardiology, Leiden University Medical Center, \\ Leiden, The Netherlands.
}

Submitted September 2012. Accepted for publication February 2013.

\begin{abstract}
The power of exercise-induced T-wave alternans (TWA) to predict the occurrence of ventricular arrhythmias was evaluated in 67 patients with an implanted cardiac defibrillator (ICD). During the 4-year follow-up, electrocardiographic (ECG) tracings were recorded in a bicycle ergometer test with increasing workload ranging from zero (NoWL) to the patient's maximal capacity (MaxWL). After the follow-up, patients were classified as either ICD_Cases $(n=29)$, if developed ventricular tachycardia/fibrillation, or ICD_Controls $(\mathrm{n}=38)$. TWA was quantified using our heart-rate adaptive match filter. Compared to NoWL, MaxWL was characterized by faster heart rates and higher TWA in both ICD_Cases $(12-18 \mu \mathrm{V}$ vs. $20-39 \mu \mathrm{V}$; P $<0.05)$ and ICD_Controls (9-15 $\mu \mathrm{V}$ vs. 20-32 $\mu \mathrm{V} ; \mathrm{P}<0.05$ ). Still, TWA was able to discriminate the two ICD groups during NoWL (sensitivity $=59-83 \%$, specificity $=53-84 \%)$ but not MaxWL (sensitivity $=55-69 \%$, specificity $=$ 39-74\%). Thus, this retrospective observational case-control study suggests that TWA's predictive power for the occurrence of ventricular arrhythmias could increase at low heart rates.
\end{abstract}

Keywords: T-wave alternans, ventricular arrhythmias, predictive power, implanted cardiac defibrillator

\section{INTRODUCTION}

T-wave alternans (TWA) is an electrophysiological phenomenon consisting in everyother-beat fluctuations of amplitude and/or shape of the electrocardiographic (ECG) Twave at stable heart rate during sinus rhythm. Macroscopic TWA is quite rare and has been identified as a harbinger of malignant ventricular arrhythmias [1]. Instead,

*Corresponding author: Laura Burattini, Polytechnic University of Marche, Department of Information Engineering, via Brecce Bianche, 60131 Ancona, Italy. Phone: (071) 220-4461. Fax: (071) 220-4220. E mail: 1.burattini@univpm.it. Other authors: S.Man@lumc.nl; C.A.Swenne@lumc.nl. 
microvolt TWA is much more common, requires specifically designed algorithms for its automatic identification [2-9], and has been recognized as a promising noninvasive index for risk stratification [10-16].

Even though microvolt TWA has been also observed in resting conditions [17, 18], it is well known that its amplitude tends to increase at high heart rates [19,20], which can be reached pharmacologically [21], by physical exercise [10, 20, 22-23] or, more invasively, by artificial pacing [21, 24, 25]. Beside increasing TWA amplitude (and, thus, the signal-to-noise ratio), fast heart rates reduce heart-rate variability [26]. Both conditions (high signal-to-noise ratio and low heart-rate variability) have been found to be fundamental for a reliable TWA identification by means of any automatic method [27], so that most risk-stratification studies have been performed under exercise conditions [10, $15,16,22-24,28,29]$. In the risk-stratification studies involving ambulatory recordings during daily activities, TWA was often measured at maximum heart rates [11, 12, 30].

Exercise-induced TWA is more easily detectable than resting TWA, but the effect of exercise on TWA predictive power for the occurrence of ventricular arrhythmias remains unknown. Thus, the aim of the present study was to evaluate if exercise, besides inducing a higher amplitude of TWA, also enhances TWA's ability to discriminate patients at higher risk for malignant ventricular arrhythmias. To this aim, exercise ECG tracings of implanted cardioverter-defibrillator (ICD) patients were analyzed. More specifically, two populations of ICD patients were considered: developing (ICD_Cases) and not developing (ICD_Controls) ventricular tachycardia or ventricular fibrillation during the 4-year follow-up. TWA's ability to discriminate the two ICD groups was evaluated at the very beginning of the exercise test when the workload was null and heart-rate was close to resting, and at the end of the exercise test when the workload matched the patient's maximum capacity and the heart rate was high.

\section{METHODS}

\subsection{Database of Exercise ECGs in Heart Failure Patients with ICDs}

The collection (from August 2006 to September 2010) of routine clinical data from 266 patients with an ICD for primary prevention because of a depressed left ventricular ejection fraction $(\mathrm{LVEF}<35 \%)$, constituting the Leiden University Medical Center (The Netherlands) database of exercise ECGs in heart failure patients with ICDs, was retrospectively selected for the present observational study on TWA. All patients underwent a 4-year follow-up starting from the date of ICD implantation. During the follow-up, patients received standard care, which included periodic visits to the outpatient clinic, amongst others, to assess validity by bicycle ergometry. The bicycle ergometer test consisted of an approximately 10-minute bicycle test during which the workload was incremented from zero (NoWL) to the patient's maximal capacity (MaxWL) by applying load-increments of $10 \%$ of the expected maximal capacity every minute. During the bicycle ergometer test, ECG recordings were obtained using a CASE 8000 stress test recorder (GE Healthcare, Freiburg, Germany; sampling frequency: 500 Hz; resolution: $4.88 \mu \mathrm{V} / \mathrm{LSB}$ ) and the 3M Red Dot ECG Electrode Soft Cloth 2271 electrodes, specifically designed for conditions where skin moisture is an issue, like stress testing. The skin was first cleaned with alcohol and abraded, to reduce electrode 
resistance. Electrode resistance was measured by the electrocardiograph, and considered acceptable if $<5 \mathrm{kOhm}$. Electrodes were applied in the Mason-Likar position.

According to the data collection protocol, during the follow-up, patients could undergo more than one exercise tests. Eventually, at the end of the follow-up, patients were classified as either "ICD_Cases" (76 patients) if, during the follow-up, they developed ventricular tachycardia or ventricular fibrillation (treated with antitachycardia pacing and/or shock therapy), or "ICD_Controls" (190 patients) otherwise. ICD_Cases exercise tests were excluded when a major cardiac event (infarction, VT ablation, coronary artery bypass graft) occurred between the exercise test and the moment of VT/VF, because ablation modified their arrhythmogenic substrate with respect to the state when the ICD was implanted. If more than one exercise test remained available for analysis, the one closest in time (either before or after) to the VT/VF episode was selected so that the substrate during the stress test would be as close as possible to the substrate during the actual arrhythmia. In ICD_Controls with more than one suitable exercise test, the earliest available one was selected. Eventually, only one ECG tracing per patient was made available for the database.

According to "Guideline for Good Clinical Practice" (European Medicines Agency, CPMP/ICH/135/95) and the data privacy law of the Netherlands, for being enrolled in the present study, which is retrospective, observational and on standard clinical data, no informed consent from patients (whose identity remained anonymous) was needed because no interventions had taken place.

\subsection{Study Population Enrollment Criteria}

Enrollment criteria were applied to screen the ICD patients in the Leiden University Medical Center database of exercise ECGs in heart failure to warrant a reliable TWA identification and a comparable clinical profile of the two ICD groups. More specifically, patients in the ICD_Cases $(\mathrm{n}=76)$ and ICD_Controls $(\mathrm{n}=190)$ groups were considered eligible for the present study if satisfying the following inclusion criteria:

- Criterion 1. The NoWL and the MaxWL phases of the exercise test were both characterized by stable heart rate. More specifically, in each phase, it had to exist at least a 64-beat ECG window characterized by $\mathrm{NN}$ standard deviation not exceeding $10 \%$ of mean NN.

- Criterion 2. ECG tracings from the NoWL and the MaxWL phases of the exercise test were both characterized by a small number of artifacts and ectopic beats in at least three of the six precordial leads (V1 to V6). More specifically, for each phase, it had to exist at least a 64-beat ECG window for which at least three leads were characterized by no more than 4 replaced beats because of artifacts and ectopic beats [27, 31].

- Criterion 3. Age, at the time of the exercise test, was between 45 and 75 years old.

- Criterion 4. LVEF, at the time of the exercise test, was $<35 \%$.

- Criterion 5. Body mass index (BMI), at the time of the exercise test, was between 20 and $34 \mathrm{Kg} / \mathrm{m}^{2}$. 


\subsection{Clinical ECG Data}

All ECG tracings were preprocessed for noise removal ( $0.5-35 \mathrm{~Hz}$ bandpass filter) and baseline subtraction by means of a third-order spline interpolation [3, 31]. Subsequently, sliding ECG windows including 64 consecutive beats were extracted every two seconds from the entire ECG recording in correspondence of the NoWL and the MaxWL phases of the exercise test, and preprocessed for artifacts and ectopic beats replacement [27, 31] before going through our heart-rate adaptive match filter procedure (see below) for TWA quantification. ECG windows characterized by unstable heart rate (NN standard deviation greater than $10 \%$ of mean) or by a number of replaced beats greater than 4 were rejected. On the contrary, ECG windows characterized by a stable heart rate and a low number $(\leq 4)$ of artifacts and ectopic beats were considered suitable for TWA analysis. These ECG suitability requirements represent an external (i.e. not integrated into the TWA algorithm) test for TWA reliability. Multiple extraction of ECG windows inside the one-minute duration of the NoWL or MaxWL phases was used to minimize the number of rejected patients, since it had to exist at least one ECG window suitable for TWA analysis inside each exercise phase for a patient not to be rejected (criterion 2). When more than a suitable window was found within a specific exercise phase, TWA measurements from all suitable windows were averaged.

\subsection{T-Wave Alternans Identification}

TWA identification was performed in a completely automatic way by individuals who were blinded to outcomes. All the 64-beat ECG windows (characterized by 6 leads each) found to be suitable for TWA analysis went through our heart-rate adaptive match filter (AMF) procedure [5] for automatic TWA identification previously validated in simulated settings $[2,27,31]$ and applied to clinical data $[5,17,18,32-$ 34]. TWA was identified in each one of the six precordial leads independently.

Ideally, at a fixed heart rate, TWA is characterized by a single frequency by definition equal to half heart rate. In our clinical 64-beat ECG windows with slowly (see enrollment criterion 1) increasing heart-rate, TWA was supposed to be characterized by a small frequency band centered in half mean (over the 64 beats) heart rate $\left(f_{T W A}\right)$. On this basis, our AMF was conceived as a heart-rate (and, thus, $f_{T W A}$ ) adaptive narrow-band passing filter (ideally a match filter) with its passing band centered in $f_{T W A}$. In our implementation, the AMF is a $6^{\text {th }}$ order bidirectional Butterworth band-pass filter characterized by a $0.12 \mathrm{~Hz}$ wide passing band centered in $f_{T W A}$, and consisted of a cascade of a low-pass filter ( $L P F$; cut-off frequency $f_{L P F}=f_{T W A}+d f_{T W A}$, with $\left.d f_{T W A}=0.06 \mathrm{~Hz}\right)$ and a high-pass filter (HPF; cut-off frequency $\left.f_{H P F}=f_{T W A}-d f_{T W A}\right)[5,31]$. The squared module of the AMF is expressed by the following equation:

$$
\left|H_{A M F}(f)\right|^{2}=\left|H_{L P F}(f)\right|^{2} \cdot\left|H_{H P F}(f)\right|^{2}=\frac{1}{1+\left(\frac{f}{f_{L P F}}\right)^{6}} \cdot \frac{\left(\frac{f}{f_{H P F}}\right)^{6}}{1+\left(\frac{f}{f_{H P F}}\right)^{6}} .
$$


Each time the AMF is fed with an ECG window which was found to be suitable for TWA analysis, it computes the ECG heart rate and the corresponding $f_{T W A}$, and filters out every ECG components, including those relative to noise but not those relative to TWA. Thus, the output of the AMF is an amplitude-modulated sinusoidal signal, i.e., the TWA signal, having the same length of the input ECG and characterized by a frequency which matches $f_{T W A}$. If really pertaining to TWA (and not, for example, to noise oscillations which match $\left.f_{T W A}\right)$, the TWA signal maxima and minima have to fall inside the JT intervals (Figure 1). Such requirement represents the internal (i.e., integrated in the TWA identification algorithm) test for a reliable TWA by the AMF. The local amplitude of the TWA signal corresponding with the $\mathrm{i}^{\text {th }}$ beat provides a quantification of the TWA amplitude (TWAA $_{\mathrm{i}}$, in $\mu \mathrm{V}$ ) characterizing that beat (Figure 1). If the TWA signal has its maxima and minima outside the repolarization segment (as in the presence of QRS alternans, for example), all TWAA $\mathrm{i}_{\mathrm{i}}$ values are set to zero. Eventually, in the case of an ECG tracing affected by no alternans of any kind, the TWA signal at the output of the AMF reduces to a zero constant signal. Consequently, all the $\mathrm{TWAA}_{\mathrm{i}}$ values are equal to zero.

The TWAA $_{i}$ values computed from each beat were averaged over the 64 beats to provide a characterization of an ECG window. (TWAA) Eventually, all the available TWAA values of the same exercise phase were averaged to obtain one TWA estimation for NoWL (TWAA_NoWL) and another TWA estimation for MaxWL (TWAA_MaxWL). For each patient, TWAA_NoWL and TWAA_MaxWL values relative to every lead were determined, together with the lead-system TWAA

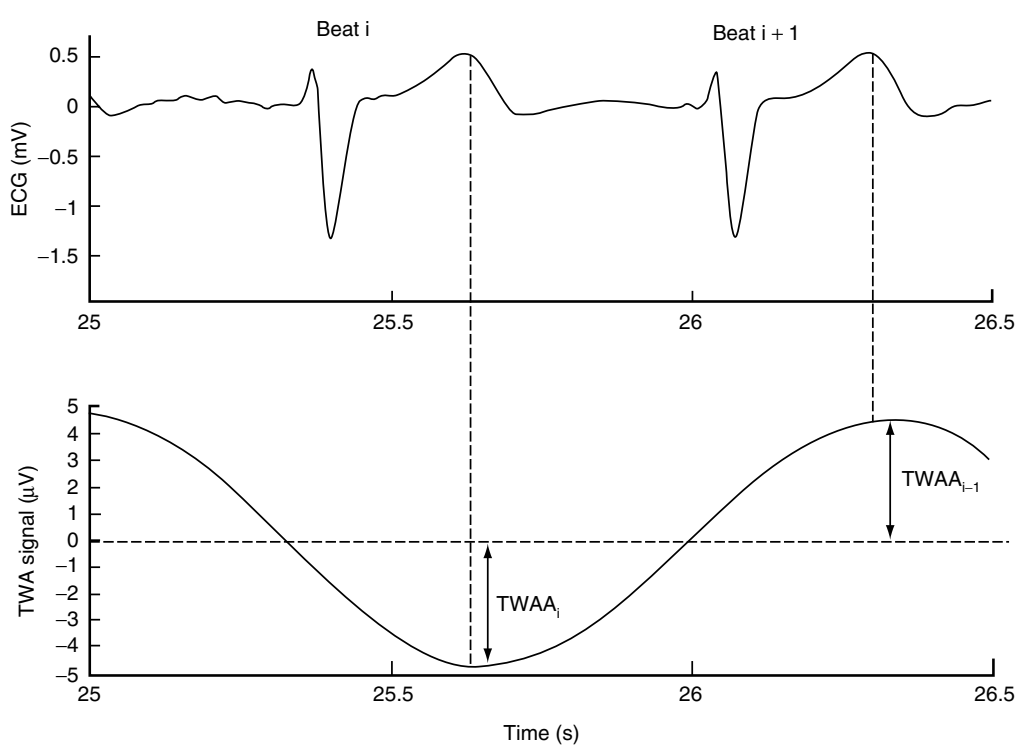

Figure 1. ECG signal (upper panel) and relative TWA signal (lower panel) at the output of the AMF from which the local TWA amplitude $\left(\right.$ TWAA $_{\mathrm{i}}$ ) corresponding with the $\mathrm{i}^{\text {th }}$ beat is estimated. 
measurements consisting of the mean and maximum TWAA values over the leads (mean V1-V6 and max V1-V6, respectively).

\subsection{Statistics}

As a consequence of screening criterion 2, some patients could have some missing TWA measurements in up to three leads. Missing data were replaced with the mean value of the corresponding population. More specifically, for each ICD group, no more than $10 \%$ of replacements (maximum 2 and 3 replacements among the ICD_Cases and ICD_Controls, respectively) were allowed in the TWAA distribution relative to a specific lead and specific phase of the exercise test.

Comparison between clinical and/or TWA parameters relative to the two ICD groups or measured during the NoWL or MaxWL phases of the exercise test were performed using parametric and non-parametric tests for normal and non-normal distributions, respectively. Normality of a parameter distribution was tested using the Lilliefors test. Comparisons between continuous and normally distributed parameters were performed using the t-test for equal means (unpaired Student's t-test for comparing the ICD_Cases against ICD_control, and the paired t-test for comparing parameters belonging to the same ICD group but measured during the NoWL vs. MaxWL phases of the exercise test), whereas comparisons between continuous and not-normally distributed parameters were performed using the Wilcoxon rank-sum test for equal medians. Eventually, differences in the binary parameters distributions between the two groups were evaluated using the chi-square test. The statistical significance level was set at $5 \%$.

To evaluate the TWA predictive power for the occurrence of ventricular arrhythmias, the receiver operating characteristic (ROC) and its area under the curve (AUC) were used. Sensitivity (Se) and specificity (Sp) values were also computed after the definition of a threshold value identified as the point where the minimum distance between $\mathrm{Se}$ and $\mathrm{Sp}$ digital curves occurred (ideally, for continuous curves, at the intersection of the ROC curve and the $\mathrm{Sp}=1$-Se line).

\section{RESULTS}

Patients screening based on the criteria in the order listed in Methods was as follows. Of the 76 ICD_Cases, 47 (62\%) patients were rejected for ECG-related reasons. More specifically, 36 patients were rejected because not characterized by stable heart rate (criterion 1 not satisfied) while the remaining 11 showed too many artifacts or ectopic beats (criterion 2 not satisfied). No patients were rejected for clinical ECG-unrelated reasons (i.e., criteria 3 to 5 always satisfied) among the ICD_Cases. On the other hand, among the 190 ICD_Controls, 94 patients were rejected because not showing stable heart rate, and 27 were rejected because showing too many artifacts and ectopic beats. Thus, globally, 121 (64\%) patients were rejected for ECG-related reasons. In addition, another $31(16 \%)$ ICD_Controls patients were rejected because of age (10 patients), LVEF (17 patients) and BMI (4 patients). Overall, 47 (62\%) ICD_Cases and 152 (80\%) ICD_Controls patients were rejected, and the final study population counted 29 ICD_Cases and 38 ICD_Controls.

Clinical parameters characterizing the two groups of ICD patients analyzed in this study are exhibited in Table 1. No statistically significant differences were observed between the ICD_Cases and ICD_Controls in terms of gender, age, body mass index, 
heart rate, therapies and medications. The ICD patients distributions over the NYHA functional classes were also comparable. LVEF was smaller for the ICD_Cases than for ICD_Controls $(28.0 \pm 10.0 \%$ vs. $32.3 \pm 7.2 \%)$.

During the NoWL phase of the exercise test, the two ICD groups were characterized by comparable heart rate (ICD_Cases: $81 \pm 10$ bpm; ICD_Controls: $79 \pm 10$ bpm; Table 1); however, compared to the ICD_Controls, the ICD_Cases showed significantly higher TWAA in all leads and lead systems (Table 2). The values of the AUC, Se and Sp quantifying the ability of TWAA to discriminate the two populations are exhibited in Table 3. Lead V6, although not characterized by the highest levels of TWAA, was the

Table 1. Clinical parameters (mean \pm standard deviation or number of occurrences) for the ICD_Cases and ICD_Controls, and P-values for comparing the two groups

\begin{tabular}{lccc}
\hline & ICD_Cases & ICD_Controls & \\
& $(\mathbf{2 9 )}$ & $(\mathbf{3 8})$ & P-value \\
\hline General: & & & \\
Age (years) & $59.1 \pm 8.5$ & $61.7 \pm 7.6$ & 0.2013 \\
Gender (male) & $22(75.9 \%)$ & $33(86.8 \%)$ & $0.20-0.30$ \\
BMI (Kg/m $\left.{ }^{2}\right)$ & $26.3 \pm 3.5$ & $26.3 \pm 3.4$ & 0.9931 \\
LVEF (\%) & $28.0 \pm 10.0$ & $32.3 \pm 7.2$ & 0.0484 \\
CRT-D & $10(34.5 \%)$ & $16(42.1 \%)$ & $0.50-0.70$ \\
NYHA functional class: & & & \\
I-II & $22(75.9 \%)$ & $30(79.0 \%)$ & $0.70-0.80$ \\
III-IV & $7(24.1 \%)$ & $8(21.1 \%)$ & $0.70-0.80$ \\
Medications: & & & \\
Beta-blocker & $28(96.6 \%)$ & $36(94.7 \%)$ & $0.70-0.80$ \\
Amiodarone & $8(27.6 \%)$ & $4(10.5 \%)$ & $0.05-0.10$ \\
Calcium antagonists & $0(0.0 \%)$ & $2(5.3 \%)$ & $0.20-0.30$ \\
Flecainide & $1(3.4 \%)$ & $0(0.0 \%)$ & $0.20-0.30$ \\
Digoxin & $2(6.9 \%)$ & $4(10.4 \%)$ & $0.50-0.70$ \\
ACE inhibitor / AT antagonist & $27(93.1 \%)$ & $37(97.4 \%)$ & $0.30-0.50$ \\
Diuretics for CHF & $22(75.8 \%)$ & $30(79.0 \%)$ & $0.70-0.80$ \\
Statins & $23(79.3 \%)$ & $32(84.2 \%)$ & $0.50-0.70$ \\
Heart rates: & & & \\
HR at rest (bpm) & $71 \pm 11$ & $70 \pm 12$ & 0.7876 \\
HR NoWL (bpm) & $81 \pm 10$ & $79 \pm 10$ & 0.5854 \\
HR MaxWL(bpm) & $122 \pm 13$ & $124 \pm 14 \%$ & 0.5009 \\
\hline
\end{tabular}

*Statistical significance $(\mathrm{P}<0.05)$.

ACE: angiotensin converting enzyme; AT: angiotensin; BMI: body mass index; CHF: congestive heart failure; CRT_D: cardiac resynchronization therapy with defibrillator; HR: heart rate; LVEF: left ventricular ejection fraction; MaxWL: maximum workload; NoWL: no workload; NYHA: New Your heart association 
lead that showed the highest ability to discriminate ICD_Cases from ICD_Controls, with an AUC of $81 \%$, a Se of $83 \%$, and a Sp of $84 \%$ (Table 3). With the only exception of V6, lead-system TWA measurements demonstrated advantages over single-lead

Table 2. TWAA median values $(\mu \mathrm{V})$ with relative $\left[2.5^{\text {th }} ; 25^{\text {th }} ; 7^{\text {th }} ; 97.5^{\text {th }}\right]$ percentiles in the two groups of ICD patients measured during the NoWL and

MaxWL phases of the exercise test, along with P-values for the comparison between the two ICD groups and for the comparison between NoWL and MaxWL measurements within a group

\begin{tabular}{|c|c|c|c|c|}
\hline & & $\begin{array}{c}\text { ICD_Cases } \\
(\mathbf{2 9})\end{array}$ & $\begin{array}{c}\text { ICD_Controls } \\
(38)\end{array}$ & $\begin{array}{c}\text { P-value } \\
\text { (cases vs. } \\
\text { controls) }\end{array}$ \\
\hline Exercise test phase & Lead & & & \\
\hline \multicolumn{5}{|l|}{ NoWL } \\
\hline & V1 & $12[6 ; 8 ; 12 ; 31]$ & $9[3 ; 7 ; 11 ; 18]$ & $0.0251^{*}$ \\
\hline & V2 & $16[6 ; 10 ; 20 ; 69]$ & $10[4 ; 7 ; 13 ; 31]$ & $0.0010^{*}$ \\
\hline & V3 & $13[5 ; 11 ; 19 ; 66]$ & $9[4 ; 7 ; 16 ; 37]$ & $0.0082 *$ \\
\hline & V4 & $14[6 ; 11 ; 18 ; 53]$ & $10[5 ; 8 ; 15 ; 46]$ & $0.0158^{*}$ \\
\hline & V5 & $18[7 ; 12 ; 18 ; 56]$ & $12[3 ; 7 ; 15 ; 51]$ & $0.0035^{*}$ \\
\hline & V6 & $17[5 ; 13 ; 17 ; 44]$ & $11[4 ; 7 ; 11 ; 34]$ & $<10^{-4 *}$ \\
\hline & Mean V1-V6 & $15[8 ; 12 ; 17 ; 48]$ & $10[5 ; 9 ; 14 ; 33]$ & $<10^{-3 *}$ \\
\hline & Max V1-V6 & 18 [13; 18; 26; 72] & $15[7 ; 11 ; 20 ; 51]$ & $<10^{-3 *}$ \\
\hline \multicolumn{5}{|l|}{ MaxWL } \\
\hline & V1 & $20[9 ; 17 ; 21 ; 43]$ & $20[5 ; 10 ; 20 ; 57]$ & 0.7223 \\
\hline & V2 & $26[6 ; 17 ; 34 ; 89]$ & $23[5 ; 14 ; 27 ; 66]$ & 0.2460 \\
\hline & V3 & $27[8 ; 13 ; 42 ; 163]$ & $23[5 ; 17 ; 31 ; 82]$ & 0.3686 \\
\hline & V4 & $27[7 ; 16 ; 31 ; 122]$ & $27[9 ; 16 ; 30 ; 115]$ & 0.5810 \\
\hline & V5 & $34[12 ; 22 ; 42 ; 86]$ & $25[8 ; 17 ; 29 ; 65]$ & $0.0071^{*}$ \\
\hline & V6 & $35[5 ; 1837 ; 104]$ & $24[8 ; 22 ; 26 ; 65]$ & 0.1398 \\
\hline & Mean V1-V6 & $26[13 ; 22 ; 36 ; 86]$ & $24[10 ; 18 ; 30 ; 56]$ & 0.1094 \\
\hline & Max V1-V6 & $39[21 ; 35 ; 58 ; 163]$ & $32[15 ; 26 ; 49 ; 115]$ & $0.0215^{*}$ \\
\hline \multicolumn{5}{|l|}{$\begin{array}{l}\text { P-value (NoWL vs. } \\
\text { MaxWL) }\end{array}$} \\
\hline & $\mathrm{V} 1$ & $<10^{-5 *}$ & $<10^{-5 *}$ & - \\
\hline & $\mathrm{V} 2$ & $0.0083 *$ & $<10^{-6 *}$ & - \\
\hline & V3 & $0.0104 *$ & $<10^{-5 *}$ & - \\
\hline & V4 & $<10^{-3 *}$ & $<10^{-7 *}$ & - \\
\hline & V5 & $<10^{-5 *}$ & $<10^{-5 *}$ & - \\
\hline & V6 & $<10^{-3 *}$ & $<10^{-9 *}$ & - \\
\hline & Mean V1-V6 & $<10^{-5 *}$ & $<10^{-8 *}$ & - \\
\hline & Max V1-V6 & $<10^{-6 *}$ & $<10^{-8 *}$ & - \\
\hline
\end{tabular}

*Statistical significance $(\mathrm{P}<0.05)$. 
analysis since they allowed discrimination between ICD groups with better values of AUC (77\% and 76\% for mean V1-V6 and max V1-V6, respectively), Se (72\% and $79 \%$ for mean V1-V6 and max V1-V6, respectively), and Sp (71\% and $68 \%$ for mean V1-V6 and max V1-V6, respectively).

During the MaxWL phase of the exercise test, the two ICD groups were also characterized by similar heart rates (ICD_Cases: $122 \pm 13$ bpm; ICD_Controls: $124 \pm 14 \mathrm{bpm}$; Table 1), which were significantly higher than those of NoWL ( $\mathrm{P}<10^{-18}$ for the ICD_Cases and $\mathrm{P}<10^{-24}$ for the ICD_Controls). In both groups, such increment in the heart rate was accompanied by a significant increment of TWAA (Table 2). However, when comparing ICD_Cases vs. ICD_Controls, significant differences were only occasionally detected (in lead V5 and max V1-V6; Table 2). Consequently, TWA's ability to discriminate the two groups was quite poor during MaxWL (V5: AUC $=69 \%$, Se $=66 \%$ and $\mathrm{Sp}=71 \%$; $\max \mathrm{V1} 1-\mathrm{V} 6$ : $\mathrm{AUC}=67 \%$, $\mathrm{Se}=69 \%$ and $\mathrm{Sp}=61 \%$ ). A comparison between the ROC curves during the NoWL (solid line) and the MaxWL (dotted line) phases of the exercise test is displayed in Figure 2.

Table 3. Area under the curve (AUC), sensitivity (Se), specificity (Sp) and TWAA thresholds for discriminating ICD_Cases from ICD_Controls during the NoWL and MaxWL phases of the exercise test

TWAA

AUC $\quad$ Se $\quad$ Sp threshold $(\mu \mathrm{V})$

Exercise test Phase

Lead

NoWL

MaxWL

$\begin{array}{cllll}\text { V1 } & 0.66 & 0.59 & 0.53 & 10 \\ \text { V2 } & 0.74 & 0.69 & 0.68 & 12 \\ \text { V3 } & 0.69 & 0.72 & 0.66 & 11 \\ \text { V4 } & 0.67 & 0.62 & 0.66 & 13 \\ \text { V5 } & 0.71 & 0.66 & 0.58 & 14 \\ \text { V6 } & 0.81 & 0.83 & 0.84 & 11 \\ \text { Mean V1-V6 } & 0.77 & 0.72 & 0.71 & 13 \\ \text { Max V1-V6 } & 0.76 & 0.79 & 0.68 & 17\end{array}$

$\begin{array}{cllll}\text { V1 } & 0.53 & 0.55 & 0.39 & 19 \\ \text { V2 } & 0.58 & 0.62 & 0.53 & 23 \\ \text { V3 } & 0.56 & 0.55 & 0.55 & 25 \\ \text { V4 } & 0.54 & 0.55 & 0.47 & 26 \\ \text { V5 } & 0.69 & 0.66 & 0.71 & 25 \\ \text { V6 } & 0.60 & 0.62 & 0.74 & 24 \\ \text { Mean V1-V6 } & 0.62 & 0.59 & 0.58 & 26 \\ \text { Max V1-V6 } & 0.67 & 0.69 & 0.61 & 35\end{array}$



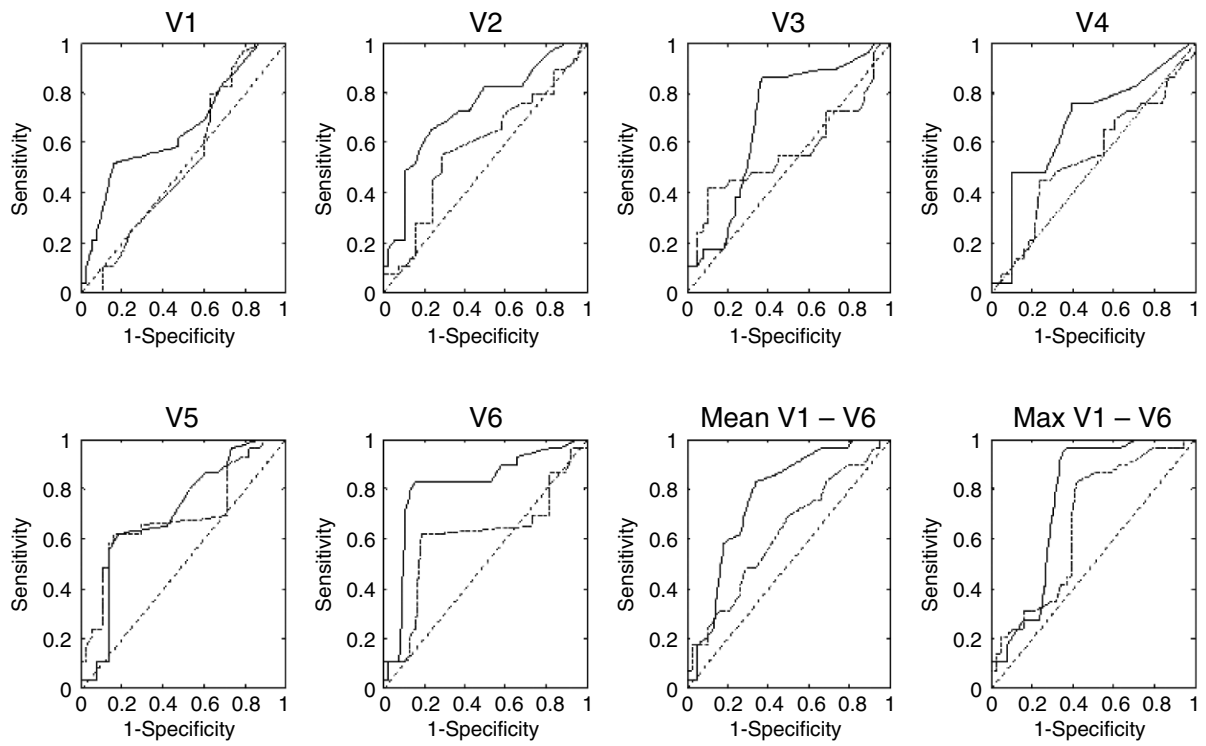

Figure 2. Receiver operating characteristics (ROC) curves for each lead or lead system during the NoWL (solid line) and the MaxWL (dotted line) phases of the exercise test.

\section{DISCUSSION}

The present study is the first to investigate if faster heart rates, reached through exercise, beside increasing TWA amplitude, also enhance the TWA's predictive power of ventricular arrhythmias. To this aim, the Leiden University Medical Center database of exercise ECGs in 266 heart failure patients with ICDs, grouped in 76 ICD_Cases (i.e. patients who developed VT/VF during follow-up) and 190 ICD_Controls (i.e. patients who did not develop ventricular arrhythmias during follow-up), was employed. Of these 266 ICD patients, 199 (75\%), corresponding to 47 ICD_Cases and 152 ICD_Controls, did not satisfied the study screening criteria and were excluded. Such high rejection rate was due to both ECG related and unrelated reasons. Being TWA a phenomenon defined at stable and sinus rhythm, ECG tracings have to be characterized by a stable heart rate and a low number of artifacts and ectopic beats [2, 3, 27] for a reliable TWA identification by any method, including our AMF. In addition, with the aim to compare TWA's predictive power during the NoWL phase vs. the MaxWL phase of the exercise test, this study required that such conditions had to be satisfied in both phases, making the enrollment criteria even more restrictive. The aforementioned ECG features required for TWA identification are hardly found in routine clinical exercise ECG recordings that are mainly for the measurement of the maximum workload and not specifically for TWA testing. Neither technology nor measurement protocol of the routine clinical exercise ECG is TWA-specific, and the levels of noise and heart-rate variability were often not acceptable. As a result, rejections due to ECG-related reasons 
were 168 (63\%, including 47 ICD_Cases and 121 ICD_Controls). The need of having two ICD groups characterized by the same clinical profile caused the rejections due to ECG-unrelated reasons, which globally counted 31 ICD patients (12\%, including 0 ICD_Cases and 31 ICD_Controls). The reported rejection rates for ECG-related vs. ECG-unrelated reasons, however, depend on the temporal order according to which the enrollment criteria were applied, as presented in Methods. By applying first criteria 3 to 5 and then criteria 1 to 2, the rejection rates due to ECG-related and ECG-unrelated reasons would likely decrease (for possible previous rejections due to clinical parameters) and increase, respectively. The final number of rejected patients, instead, is independent of the criteria application order. Eventually, 29 (38\%) ICD_Cases and 38 (20\%) ICD_Controls were found to be qualified for the present study.

Although the ECG tracings of the enrolled patients underwent preprocessing for noise filtering and baseline subtraction, and were found to be suitable for TWA analysis, a small heart-rate variability and a low level of noise could still affect them. Consequently, automatic TWA identification was performed using our AMF method, which, compared to several other techniques, proved to be particularly robust to the presence of such interferences [27]. AMF robustness to noise is due to the fact that, each time the AMF is fed with a suitable ECG, it computes the ECG heart rate and, thus the corresponding TWA frequency, and filters out all ECG components including those related to noise, but not those related to TWA. Still, the presence of noise characterized by a frequency band overlapping $f_{T W A}$ could jeopardize TWA estimation. Indeed, the noise component at $f_{T W A}$ will survive preprocessing, corrupt the TWA signal determination and, consequently, ruin the TWAA estimation. Muscular noise and motion noise are two very common types of noise affecting real ECGs and characterized by a wide frequency band possibly overlapping $f_{T W A}$. However, their presence often causes artifacts in the ECG tracing whose identification in the pre-processing stage should lead to the tracing rejection $[2,3,27]$.

In our study, pedaling frequency could also be an issue. Indeed, if $f_{T W A}$ is an integer multiple of the pedaling frequency, the noise will have (according to the Fourier's analysis) a frequency component matching TWA frequency. For this reason, exercise tests specifically designed for TWA analysis should keep pedaling frequency under control. In the present retrospective study, we used exercise data in which pedaling frequency was not controlled since, as mentioned before, these data consisted of routine clinical exercise ECG recordings not specifically for TWA testing. However, in the presence of this rather unlikely but still possible situation of a pedaling noise component matching the TWA frequency, TWA would not be identified by the AMF unless the noise component (i.e. sinusoid) at the TWA frequency also matches TWA phase. In fact, to warrant a reliable TWA identification, the maxima and the minima of the sinusoidal signal at the output of the AMF must occur in correspondence of the JT segment for TWA to be identified. Only in the case of a noise component of the same frequency and phase of TWA, the noise would be falsely recognized as TWA by the AMF. Thus, for what concerns the presence of noise, while it is true that muscular, motion or pedaling noise could affect TWA measurements, the occasional occurrence of such cases should result in a limited statistical relevance. AMF robustness to subtle 
heart-rate variability is due to the fact that, in real clinical cases, TWA is assumed to be characterized not only by a single frequency at half heart rate, as it would ideally be at fixed heart rate, but also by a small frequency band centered in $f_{T W A}$ (i.e., mean half heart rate). These features of the AMF method make it a reliable technique to detect TWA in both rest and exercise conditions. To limit the noise propagation and the number of computations, the AMF was applied only to the six precordial leads, which were found to be particularly suitable for TWA identification in these [33] and other [28-30, 35, 36] groups of patients.

TWA may occur as a regionally specific phenomenon [35, 37] and can be lead-dependent. For this reason, a TWA value was provided for each one of the 6 precordial leads, as previously found to be particularly suitable for TWA identification in ICD patients [33]. In addition, lead-system (mean V1-V6 and max V1-V6) TWA values were also provided, and proved to have a greater ability to discriminate the two groups of ICD patients than most of the single lead measurements. However, mean V1-V6 and/or max V1-V6 TWAA values provide no insights into the spatial localization of TWA in the heart. By definition, max V1-V6 TWA may be more significantly affected by noise than mean V1-V6 TWA; therefore, the latter is supposed to provide a more robust TWA estimation. On the other hand, max V1-V6 TWA is more likely to be able to detect spatially specific TWA episodes which, on the surface ECG, will appear only in a few leads and would remain hidden by the averaging procedure.

During the NoWL phase of the exercise test, heart rates were similar in the two ICD groups (about $80 \mathrm{bpm}$ ). Nevertheless, TWA was quite low but significantly higher in the ICD_Cases $(12-18 \mu \mathrm{V})$ than in the ICD_Controls $(9-15 \mu \mathrm{V})$. This finding was observed in all leads and lead system measurements, even though V6 appeared to be the most powerful for discrimination of the two ICD groups, and therefore, for TWA's prediction of ventricular arrhythmias $(\mathrm{Se}=83 \%, \mathrm{Sp}=84 \%)$. During the MaxWL phase of the exercise test, heart rates remained similar in the two ICD groups (around 123 bpm) and, as expected, was significantly higher than during NoWL. Accordingly, TWAA increased significantly in both the ICD_Cases (20-39 $\mu \mathrm{V})$ and ICD_Controls (20-32 $\mu \mathrm{V})$. Such finding, by confirming the well known TWA dependency on heart rate [20], also validates the AMF's ability to provide reliable TWA measurements in both resting and exercise conditions. Even though significantly higher values of TWA were observed in all leads and lead-system measurements, when comparing ICD_Cases vs. ICD_Controls, statistical significance was found only for V5 and max V1-V6 measurements (with values of Se for V5 being 66\% and max V1-V6 being 69\%; and values for Sp for V5 being 71\% and max V1-V6 being 61\%), which made TWA's ability to discriminate the two ICD groups practically negligible during MaxWL. Thus, in our ICD patients, AMF-derived TWA ability to discriminate patients at risk for malignant ventricular arrhythmias is higher at lower heart rates where TWAA is also smaller, than during exercise where both heart rate and TWA are higher.

Several studies have shown that exercise-induced TWA is capable of stratifying risk for cardiovascular death and lethal arrhythmias [10, 15, 16, 22-24, 28, 29]. However, other studies have not confirmed the usefulness of TWA in risk stratification [38, 39], but rather conclude that the TWA related benefits seem to vary with studied population, so that further investigations are needed before TWA's incremental prognostic role can 
be properly defined [40]. In a recent document providing a consensus guideline for TWA, authors conclude that even though it is reasonable to consider TWA evaluation whenever there is suspicion of vulnerability to lethal cardiac arrhythmias, there is as yet no definite evidence from interventional trials that it can guide to therapy [41]. Our results simply suggest that TWA at rest, though having lower amplitude, could discriminate better than TWA during exercise, when its amplitude is larger. Such finding, which specifically relates to our AMF method, is not in contrast with the aforementioned studies on exercise TWA performed using other techniques. Indeed, it does not imply that TWA has no predicting power when measured during exercise, but rather, invite new evaluations, eventually performed using other methods, on the TWA predictive power at rest. Other works have also suggested that TWA is more specific when measured at low heart rates, but it is also less sensitive, while at frequencies higher than 110-120 bpm, it has a good sensitivity but a poor specificity [42, 43]. In our study, we found that both sensitivity and specificity are better during the NoWL phase of the exercise test when heart rate is lower, than during the MaxWL when heart rate is higher. Such a difference from the previous studies is probably due to the different axis along which the study is performed. Indeed, our axis is the workload, not the HR, even though the two quantities are clearly correlated. Still, low HR to us means $81 \pm 10 \mathrm{bpm}$ and 79 $\pm 10 \mathrm{bpm}$ for ICD_Cases and ICD_Controls, respectively, and not exactly 90 $\mathrm{bpm}$. Analogously, high HR to us means $122 \pm 13 \mathrm{bpm}$ and $124 \pm 14 \mathrm{bpm}$, and not exactly $120 \mathrm{bpm}$. In other words, our low and high heart-rate values are characterized by a significant variability due to their patients' dependency. Thus, if Tanno et al. [42] and Kitamura et al. [43] studied TWA as a function of frequency, our study is an analysis of TWA in resting vs. exercise conditions, respectively, approximated and defined as the NoWL and the MaxWL phases of the exercise test. Eventually, the possibility of TWA's predictive power of ventricular arrhythmias being higher at rest than under exercise also parallels the observation that several characteristics (such as QRS width and QRS-T spatial angle) have been identified in ordinary $10 \mathrm{~s}$ resting ECGs to also have predictive value [44-46]. Nevertheless, given the small but still representative number of the enrolled patients, which represents the major limitation of the present study, future analysis on ECG recordings specifically performed for TWA testing (to reduce the number of rejected patients) are desirable. More specifically, special TWA electrodes (e.g., the Micro-V Alternans Sensors proposed by the Cambridge Heart, usually used for TWA studies performed by the standardized spectral method) and measurement protocols in which heart rate is kept constant during the NoWL and MaxWL phases of the exercise test, together with TWA quantification performed by other methods, should be considered. Optimization of ECG recordings for TWA testing, however, was beyond the scope of the present work, in which ECG signal quality has been the major cause of exclusions.

\section{CONCLUSION}

In our 66 ICD patients, increments in the heart rates, reached through exercise, are accompanied not only by an increase in the TWA amplitude but also by a decrease in the TWA's ability to discriminate ICD_Cases from ICD_Controls. The present study, performed using our AMF-method, suggests that TWA's predictive power for ventricular 
arrhythmias could be better at low heart rates (when TWA amplitude is smaller) than at high heart rates (when TWA amplitude is higher). However, given the relatively small number of patients involved in the study and the data quality, future studies possibly performed on better data and using other methods, are needed to confirm our findings.

\section{ACKNOWLEDGEMENTS}

This study was supported by the Netherlands Heart Foundation.

\section{CONFLICT OF INTEREST}

All authors have no financial or personal relationships with other people or organizations that could inappropriately influence (bias) this work.

\section{NOMENCLATURE}

$\begin{array}{ll}\text { ACE } & \text { Angiotensin Converting Enzyme } \\ \text { AMF } & \text { Adaptive Match Filter } \\ \text { AT } & \text { Angiotensin } \\ \text { AUC } & \text { Area Under the Curve } \\ \text { BMI } & \text { Body Mass Index }\left(\mathrm{Kg} / \mathrm{m}^{2}\right) \\ \text { CHF } & \text { Congestive Heart Failure } \\ \text { CRT_D } & \text { Cardiac Resynchronization Therapy with Defibrillator } \\ \text { ECG } & \text { Electrocardiogram } \\ \text { HR } & \text { Heart Rate (bpm) } \\ \text { ICD } & \text { Implanted Cardiac Defibrillator } \\ \text { LVEF } & \text { Left Ventricular Ejection Fraction, \% } \\ \text { MaxWL } & \text { Maximum Work Load } \\ \text { NoWL } & \text { No Work Load } \\ \text { NYHA } & \text { New Your Heart Association } \\ \text { ROC } & \text { Receiver Operating Characteristic } \\ \text { Se } & \text { Sensitivity } \\ \text { Sp } & \text { Specificity } \\ \text { TWA } & \text { T-Wave Alternans } \\ \text { TWAA } & \text { T-Wave Alternans amplitude, } \mu \text { V } \\ \text { VF } & \text { Ventricular Tachicardia } \\ \text { VT } & \text { Ventricular Fibrillation }\end{array}$

\section{REFERENCES}

[1] Raeder EA, Rosenbaum DS, Bhasin R, Cohen RJ. Alternating morphology of the QRST complex preceding sudden death. The New England Journal of Medicine, 1992, 326(4):271-272.

[2] Burattini L, Bini S, Burattini R. Comparative analysis of methods for automatic detection and quantification of microvolt T-wave alternans. Medical Engineering \& Physics, 2009, 31(10):1290-1298.

[3] Martínez JP, Olmos S. Methodological principles of T wave alternans analysis: a unified framework. IEEE Transactions on Bio-Medical Engineering, 2005, 52(4):599-613.

[4] Martínez JP, Olmos S, Wagner G, Laguna P. Characterization of T-wave alternans during ischemia: time-course and spatial analysis. IEEE Transactions on Bio-Medical Engineering, 2006, 53(4):701-711. 
[5] Burattini L, Zareba W, Burattini R. Adaptive match filter based method for time vs. amplitude characterization of microvolt ECG T-wave alternans. Annals of Biomedical Engineering, 2008, 36(9):1558-1564.

[6] Smith JM, Clancy EA, Valeri CR, Ruskin JN, Cohen RJ. Electrical alternans and cardiac electrical instability. Circulation, 1988, 77(1):110-121.

[7] Nearing BD, Huang AH, Verrier RL. Dynamic tracking of cardiac vulnerability by complex demodulation of the T wave. Science, 1991, 252(5004):437-440.

[8] Nearing BD, Verrier RL. Modified moving average analysis of T-wave alternans to predict ventricular fibrillation with high accuracy. Journal of Applied Physiology, 2002, 92(2):541-549.

[9] Burattini L, Zareba W, Moss AJ. Correlation method for detection of transient T-wave alternans in digital ECG recordings. Annals of Noninvasive Electrocardiology, 1999, 4(4):416-424.

[10] Leino J, Minkkinen M, Nieminen T, Lehtimäki T, Viik J, Lehtinen R, Nikus K, Kööbi T, Turjanmaa V, Verrier RL, Kähönen M. Combined assessment of heart rate recovery and T-wave alternans during routine exercise testing improves prediction of total and cardiovascular mortality: the Finnish Cardiovascular Study. Heart Rhythm, 2009, 6(12):1765-1771.

[11] Maeda S, Nishizaki M, Yamawake N, Ashikaga T, Shimada H, Asano M, Ihara K, Murai T, Suzuki H, Fujii H, Sakurada H, Hiraoka M, Isobe M. Ambulatory ECG-based T-wave alternans and heart rate turbulence predict high risk of arrhythmic events in patients with old myocardial infarction. Circulation Journal, 2009, 73(12):2223-2228.

[12] Sakaki K, Ikeda T, Miwa Y, Miyakoshi M, Abe A, Tsukada T, Ishiguro H, Mera H, Yusu S, Yoshino H. Time-domain T-wave alternans measured from Holter electrocardiograms predicts cardiac mortality in patients with left ventricular dysfunction: a prospective study. Heart Rhythm, 2009, 6(3):332-337.

[13] Hohnloser SH. T-wave alternans: a pathophysiological link to human ventricular tachyarrhythmias. Heart Rhythm, 2008, 5(5):677-678.

[14] Narayan SM. T-wave alternans and the susceptibility to ventricular arrhythmias. Journal of the American College of Cardiology, 2006, 47(2):269-281.

[15] Ikeda T, Yoshino H, Sugi K, Tanno K, Shimizu H, Watanabe J, Kasamaki Y, Yoshida A, Kato T. Predictive value of microvolt T-wave alternans for sudden cardiac death in patients with preserved cardiac function after acute myocardial infarction: results of a collaborative cohort study. Journal of the American College of Cardiology, 2006, 48(11):2268-2274.

[16] Klingenheben T, Zabel M, D’Agostino RB, Cohen RJ, Hohnloser SH. Predictive value of T-wave alternans for arrhythmic events in patients with congestive heart failure. Lancet, 2000, 356(9230):651-652.

[17] Burattini L, Zareba W, R Burattini. Assessment of physiological amplitude, duration and magnitude of ECG T-wave alternans. Annals of Noninvasive Electrocardiology, 2009, 14(4):366-374.

[18] L Burattini, W Zareba, R Burattini. Is T-wave alternans T-wave amplitude dependent? Biomedical Signal Processing and Control, 2012, 7(4):358-364.

[19] Narayan SM, Smith JM. Differing rate dependence and temporal distribution of T-wave alternans in patients with and without ventricular tachycardia. Journal of Cardiovascular Electrophysiology, 1999, 10(1):61-71.

[20] Bloomfield DM, Hohnloser SH, Cohen RJ. Interpretation and classification of microvolt T-wave alternans tests. Journal of Cardiovascular Electrophysiology, 2002, 13(5):502-512.

[21] Weber S, Schwab JO. Comparison of microvolt T-wave alternans measurements using atrial pacing compared to atropine administration. Pacing and clinical electrophysiology, 2007, 30(12):1487-1492.

[22] Gold MR, Bloomfield DM, Anderson KP, El-Sherif NE, Wilber DJ, Groh WJ, Estes NA 3rd, Kaufman ES, Greenberg ML, Rosenbaum DS. A comparison of T-wave alternans, signal averaged electrocardiography and programmed ventricular stimulation for arrhythmia risk stratification. Journal of the American College of Cardiology, 2000, 36(7):2247-2253.

[23] Rashba EJ, Osman AF, MacMurdy K, Kirk MM, Sarang S, Peters RW, Shorofsky SR, Gold MR. Exercise is superior to pacing for T-wave alternans measurement in subjects with chronic coronary 
artery disease and left ventricular dysfunction. Journal of Cardiovascular Electrophysiology, 2002, 13(9):845-850.

[24] Rosenbaum DS, Jackson LE, Smith JM, Garan H, Ruskin JN, Cohen RJ. Electrical alternans and vulnerability to ventricular arrhythmias. The New England Journal of Medicine, 1994, 330(4):235-241.

[25] Narayan SM, Smith JM, Cain ME. T-wave alternans using ventricular and atrial pacing. Pacing and Clinical Electrophysiology, 2005, 28 (10):1145-1146.

[26] de la Cruz Torres B, López López C, Naranjo Orellana J. Analysis of heart rate variability at rest and during aerobic exercise: a study in healthy people and cardiac patients. British Journal of Sports Medicine, 2008, 42(9):715-20.

[27] Burattini L, Bini S, Burattini R. Automatic microvolt T-wave alternans identification in relation to ECG interferences surviving preprocessing. Medical Engineering \& Physics, 2011, 33(1):17-30.

[28] Klingenheben T, Ptaszynski P, Hohnloser SH. Quantitative assessment of microvolt T-wave alternans in patients with congestive heart failure. Journal of Cardiovascular Electrophysiology, 2005, 16(6):620-624.

[29] Minkkinen M, Kähönen M, Viik J, Nikus K, Lehtimäki T, Lehtinen R, Kööbi T, Turjanmaa V, Kaiser W, Verrier RL, Nieminen T. Enhanced predictive power of quantitative TWA during routine exercise testing in the Finnish Cardiovascular Study. Journal of Cardiovascular Electrophysiology, 2009, 20(4):408-415.

[30] Verrier RL, Nearing BD, La Rovere MT, Pinna GD, Mittleman MA, Bigger JT Jr, Schwartz PJ, ATRAMI Investigators. Ambulatory electrocardiogram-based tracking of T-wave alternans in postmyocardial infarction patients to assess risk of cardiac arrest or arrhythmic death. Journal of Cardiovascular Electrophysiology, 2003, 14(7):705-711.

[31] Burattini L, Zareba W, Burattini R. Automatic detection of microvolt T-wave alternans in Holter recordings: Effect of baseline wandering. Biomedical Signal Processing and Control, 2006, 1(2):162-168.

[32] Burattini L, Bini S, Burattini R. Repolarization alternans heterogeneity in healthy subjects and acute myocardial infarction patients. Medical Engineering \& Physics, 2012, 34(3):305-312.

[33] Burattini L, Man S, Burattini R, Swenne CA. Comparison of standard vs. orthogonal ECG leads for T-wave alternans identification. Annals of Noninvasive Electrocardiology, 2012, 17(2):130-140.

[34] Man SC, De Winter PV, Maan AC, Thijssen J, Borleffs JW, van Meerwijk WP, Bootsma M, van Erven L, van der Wall EE, Schalij MJ, Burattini L, Burattini R, Swenne CA. Predictive Power of T-wave Alternans and of Ventricular Gradient 2 Hysteresis for the Occurrence of Ventricular Arrhythmias in Primary Prevention ICD Patients. Journal of Electrocardioliology, 2011, 44(5):453-459.

[35] Nearing BD, Oesterle SN, Verrier RL. Quantification of ischaemia induced vulnerability by precordial T-wave alternans analysis in dog and human. Cardiovascular Research, 1994, 28(9):1440-1449.

[36] Ruta J, Strumillo P. Usefulness of the Poincaré maps in detection of T-wave alternans in precordial leads of standard ECG: A comparison with the spectral method. Medical Science Monitor: International Medical Journal of Experimental and Clinical Research, 2001, 7(3):471-476.

[37] Salerno JA, Previtali M, Panciroli C, Klersy C, Chimienti M, Regazzi Bonora M, Marangoni E, Falcone C, Guasti L, Campana C, et al.Ventricular arrhythmias during acute myocardial ischaemia in man. The role and significance of R-ST-T alternans and the prevention of ischaemic sudden death by medical treatment. European Heart Journal, 1986,7:63-75.

[38] Gupta A, Hoang DD, Karliner L, Tice JA, Heidenreich P, Wang PJ, Turakhia MP. Ability of microvolt T-wave alternans to modify risk assessment of ventricular tachyarrhythmic events: a meta-analysis. American Heart Journal, 2012,163(3):354-364.

[39] Gehi AK, Stein RH, Metz LD, Gomes JA. Microvolt T-wave alternans for the risk stratification of ventricular tachyarrhythmic events: a meta-analysis. Journal of the American College of Cardiology, 2005, 46(1):75-82.

[40] Myles RC, Jackson CE, Tsorlalis I, Petrie MC, McMurray JJ, Cobbe SM. Is microvolt T-wave alternans the answer to risk stratification in heart failure? Circulation, 2007, 116(25):2984-2991. 
[41] Verrier RL, Klingenheben T, Malik M, El-Sherif N, Exner D, Hohnloser S, Ikeda T, Martinez JP, Narayan S, Nieminen T, Rosenbaum DS. Microvolt T-wave alternans: Physiologic basis, methods of measurement, and clinical utility. Consensus guideline by the International Society for Holter and Noninvasive Electrocardiology. Journal of the American College of Cardiology, 2011, 58(13):1309-1324.

[42] Tanno K, Ryu S, Watanabe N, Minoura Y, Kawamura M, Asano T, Kobayashi Y, Katagiri T. Microvolt T-wave alternans as a predictor of ventricular tachyarrhythmias. A prospective study using atrial pacing. Circulation, 2004, 109:1854-1858.

[43] Kitamura H, Ohnishi Y, Okajima K, Ishida A, Galeano E, Adachi K, Yokoyama M. Onset heart rate of microvolt-level T-wave alternans provides clinical and prognostic value in nonischemic dilated cardiomyopathy. Journal of the American College of Cardiology, 2002, 39(2):295-300.

[44] Barsheshet A, Moss AJ, Huang DT, McNitt S, Zareba W, Goldenberg I. Applicability of a risk score for prediction of the long-term (8-year) benefit of the implantable cardioverter-defibrillator. Journal of the American College of Cardiology, 2012, 59(23):2075-2079.

[45] Jiménez-Candil J, Ruiz M, Herrero J, León V, Martín A, Moriñigo J, Ledesma C, Martín-Luengo C. Relationship between the duration of the basal QRS complex and electrical therapies for ventricular tachycardias among ICD patients. Pacing and Clinical Electrophysiology, 2010, 33(5):596-604.

[46] Borleffs CJ, Scherptong RW, Man SC, van Welsenes GH, Bax JJ, van Erven L, Swenne CA, Schalij MJ. Predicting ventricular arrhythmias in patients with ischemic heart disease: clinical application of the ECG-derived QRS-T angle. Circulation. Arrhythmia and Electrophysiology, 2009, 2(5):548-554. 



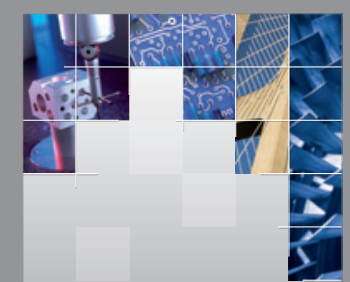

\section{Enfincering}
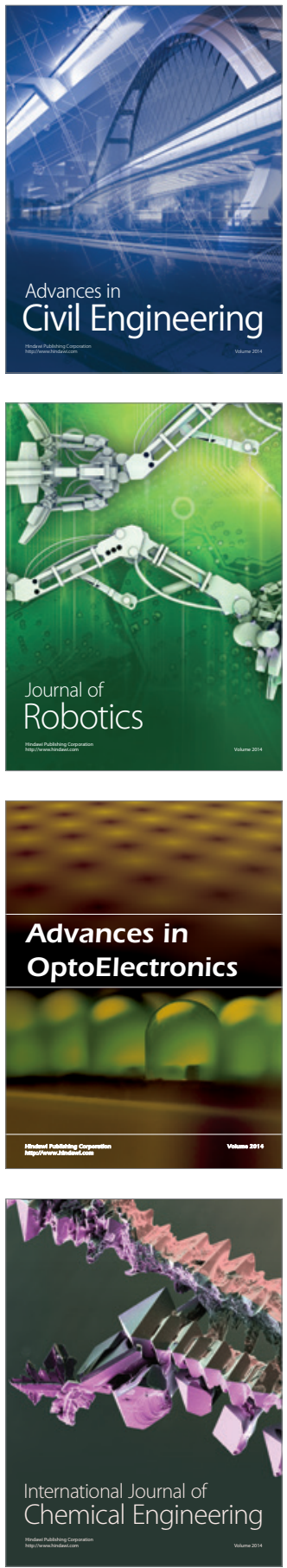

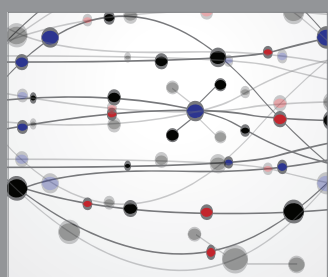

The Scientific World Journal

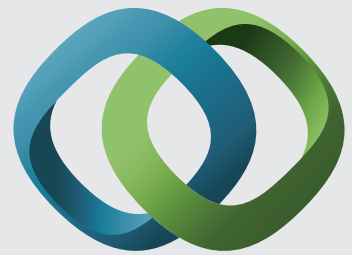

\section{Hindawi}

Submit your manuscripts at

http://www.hindawi.com
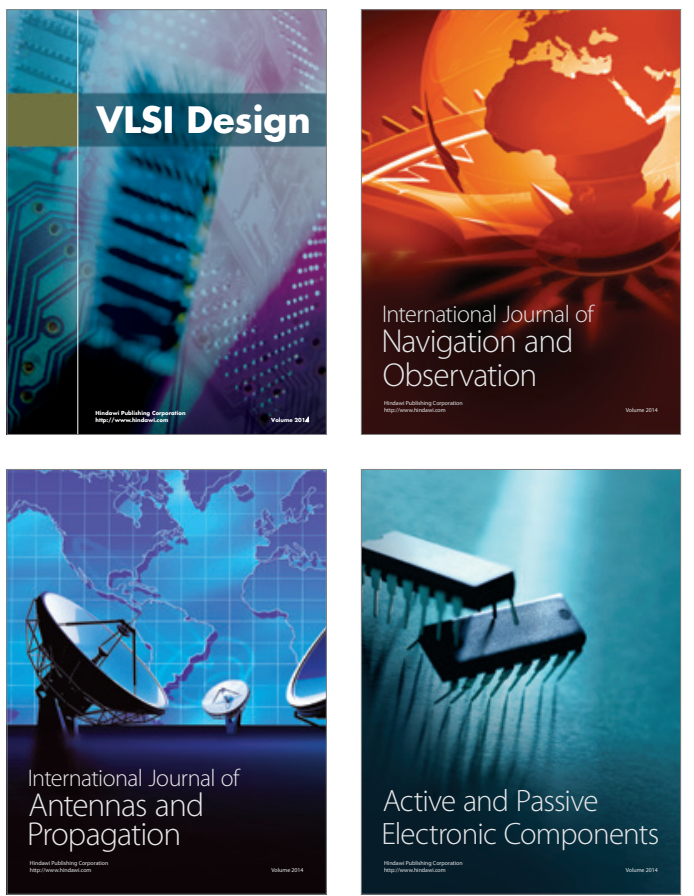
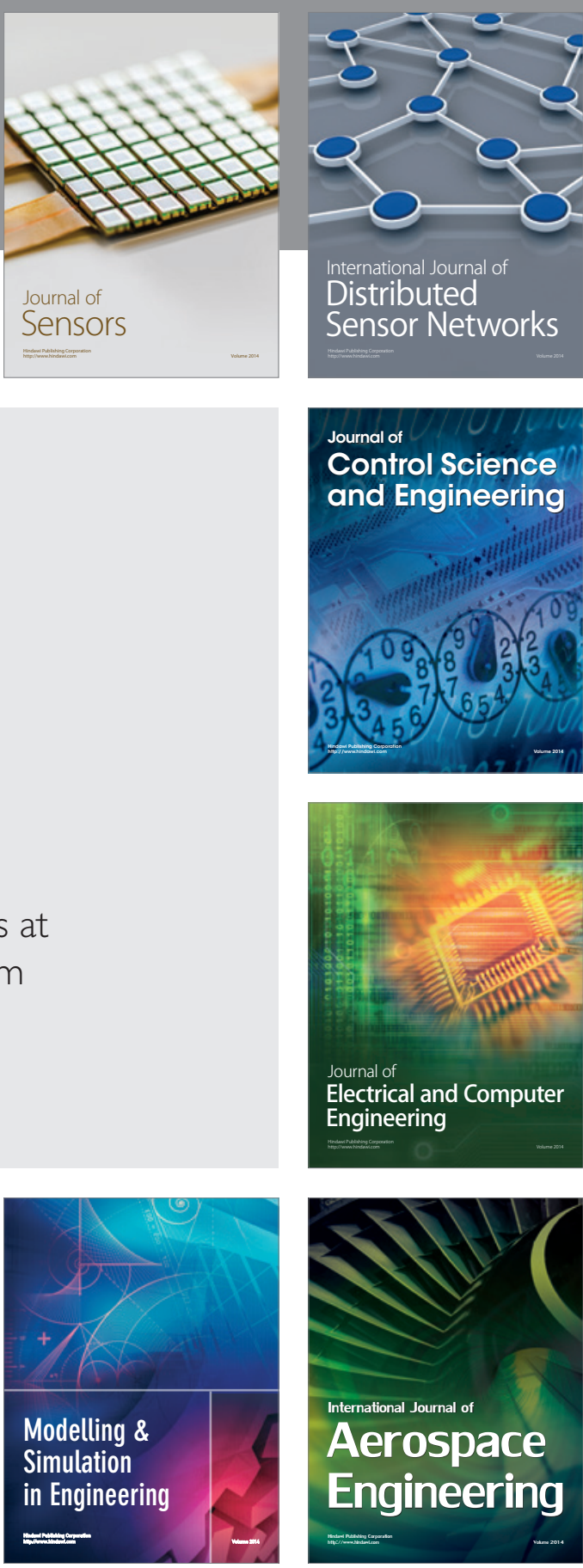

International Journal of

Distributed

Sensor Networks

Journal of

Control Science

and Engineering
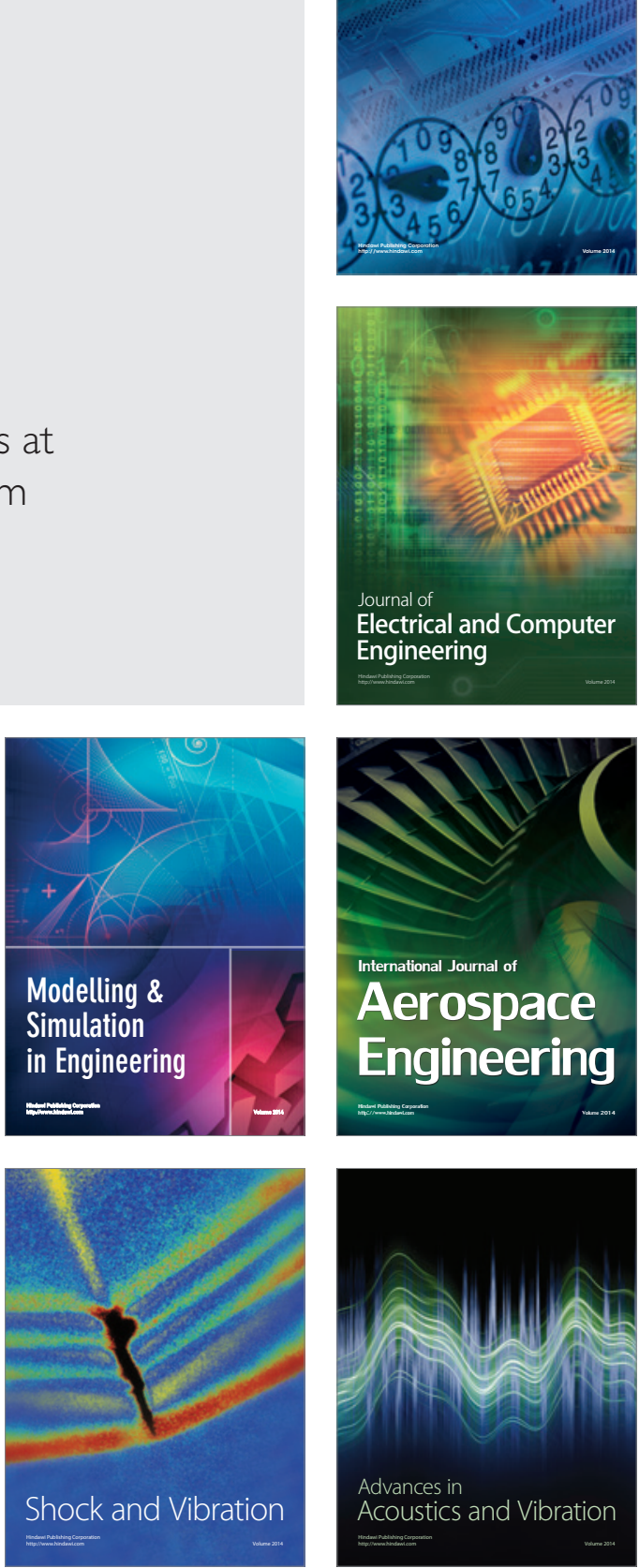